Int. J. Morphol.,

32(2):571-574, 2014

\title{
Método de Recuperación para Piezas Anatómicas del Sistema Nervioso Central
}

\author{
Recovery Method for Anatomical Pieces from the Central Nervous System
}

Marcos Valenzuela Oróstica \& Reinaldo Soto Norambuena

VALENZUELA, O. M. \& SOTO, N. R. Método de recuperación para piezas anatómicas del sistema nervioso central. Int. J. Morphol., 32(2):571-574, 2014.

RESUMEN: El material anatómico del sistema nervioso central es cada vez más difícil de obtener. A pesar de estar fijado, es muy lábil y al ser expuesto a diversas condiciones ambientales durante las actividades docentes, se va deteriorando, resecándose, adquiriendo una consistencia rígida y un aspecto oscuro que finalmente hace difícil el reconocimiento de estructuras. De la misma manera, cuando obtenemos una pieza de un cadáver con larga data de fallecido, nos podemos encontrar con un encéfalo que por lo reseco de su estado, no presta mayor utilidad. El objetivo de esta técnica es recuperar estas muestras, para que puedan ser utilizadas convenientemente en el estudio anatómico. Se usaron distintos segmentos de encéfalo, incluidos algunos contaminados por hongos y otros obtenidos de cadáveres antiguos. Los materiales utilizados fueron, agua oxigenada, agua destilada, formalina y recipientes plásticos. Se comienza limpiando manualmente las muestras de restos de polvo y cuerpos extraños que se encuentren en su superficie. Se continúa con baños en agua oxigenada, intercalando con rehidrataciones en agua destilada, hasta obtener el color y textura deseados que permitan distinguir macroscópicamente estructuras de la muestra. Posteriormente se refuerza la fijación sumergiéndolas en formalina, para luego conservarlas en forma indefinida, humedecidas con este fijador en bolsas plásticas selladas y dentro de caja plásticas tapadas. Otras muestras fueron plastinadas posteriormente. Al finalizar la técnica la mayoría de las muestras se recuperaron notoriamente, permitiendo reconocer estructuras que por su deterioro era imposible apreciar con anterioridad. En conclusión, este método permite recuperar y darle uso a muestras que estaban prácticamente desechadas.

PALABRAS CLAVE: Muestras de Sistema Nervioso Central; Agua oxigenada; Rehidratación; Recuperación.

\section{INTRODUCCIÓN}

El material anatómico en general es escaso y cada vez más difícil de obtener. Si hablamos de piezas del sistema nervioso central lo es más aún, debiendo necesariamente preservarlos mediante aplicación de sustancias químicas que detengan el proceso de autolisis (Bertone et al., 2011; Wolff et al., 2012).

Existen técnicas como la Plastinación (Valdés et al., 2010; Valenzuela et al., 2012), que permiten conservar muestras en excelente estado y de manera indefinida, pero esto no siempre es posible, principalmente por su complejidad y costo.

El tejido nervioso es muy lábil, y a pesar de estar fijado, al ser expuesto a variadas condiciones ambientales se va deteriorando, resecándose, adquiriendo una consistencia rígida y aspecto oscuro e incluso pudiendo llegar a ser contaminados por hongos, lo que hace difícil reconocer estructuras. De la misma manera, cuando obtenemos la pieza de un cadáver con larga data de fallecido, nos podemos en- contrar con un encéfalo, que por su estado reseco y oscuro, no presta utilidad alguna. El objetivo de este estudio fue elaborar una técnica que permita recuperar muestras de tejido nervioso central, que han sido rechazadas como material docente por su deterioro, mediante el uso de reactivos de fácil manejo, bajo costo y que lo haga accesible a cualquier laboratorio de anatomía.

\section{MATERIAL Y MÉTODO}

Se usaron tres tipos de muestras: (A) Segmentos de encéfalo resecos y de un color café oscuro por su exposición y uso prolongado (Fig. 1), (B) cortes de encéfalo obtenidos directamente de un cadáver con larga data de fallecido resecas y negras (Fig. 2a y 2b) y (C) encéfalos contaminados por hongos (Fig. 3). Para cada uno de ellos se realizó un protocolo de recuperación. 
A.- Muestras resecas y opacas por factores ambientales:

1. Baño en agua oxigenada de 20 volúmenes por $4 \mathrm{~h}$.

2. Rehidratación en agua destilada por $15 \mathrm{~h}$.

3. Segundo baño en agua oxigenada de 20 volúmenes por $3 \mathrm{~h}$.

4. Segundo período de rehidratación con agua destilada por $4 \mathrm{~h}$.

5. Reforzamiento de la fijación con formalina acuosa al $10 \%$ por 5 días.

6. Conservación de algunas muestras en bolsas plásticas con cierre hermético por tiempo indefinido, mientras que otras fueron plastinadas.

B.- Muestras obtenidas de cadáver antiguo:

1. Baño en agua oxigenada de 30 volúmenes por $6 \mathrm{~h}$.

2. Rehidratación en agua destilada por $24 \mathrm{~h}$.

3. Segundo baño en agua oxigenada de 20 volúmenes por $24 \mathrm{~h}$.

4. Segundo período de rehidratación con agua destilada por $24 \mathrm{~h}$.

5. Tercer baño en agua oxigenada de 20 volúmenes por $2 \mathrm{~h}$.

6. Tercer período de rehidratación con agua destilada por $6 \mathrm{~h}$.

7. Reforzamiento de la fijación con formalina acuosa al $10 \%$ por 10 días.

8. Se colocan las muestras envueltas en toallas desechables impregnadas con formalina acuosa al $5 \%$, dentro de bolsas plásticas con cierre hermético.

C.- Muestras contaminadas con hongos:

1. Limpieza en seco con pinzas anatómicas y bisturís de hojas fijas y punta roma.

2. Baño en agua oxigenada de 20 volúmenes por $24 \mathrm{~h}$.

3. Lavado rápido en agua corriente más limpieza con bisturí para sacar restos de hongos.

4. Reforzamiento de la fijación con formalina acuosa al $10 \%$ por $48 \mathrm{~h}$.

5. Segundo baño en agua oxigenada de 20 volúmenes por $24 \mathrm{~h}$.

6. Inmersión en formalina al $10 \%$ por tiempo indefinido.

\section{RESULTADOS}

La mayoría de las muestras se recuperaron notoriamente, y se reconocen estructuras que antes del procedimiento respectivo, por su deterioro, era imposibles apreciar (Figs. 4, 5 y 6). Particularmente, las muestras de los grupos A y B, recuperan color, textura y humedad (Figs. 4, 5a y $5 b)$. Se probó rehidratar algunas muestras de los grupos A y B con agua corriente no obteniendo resultados óptimos.

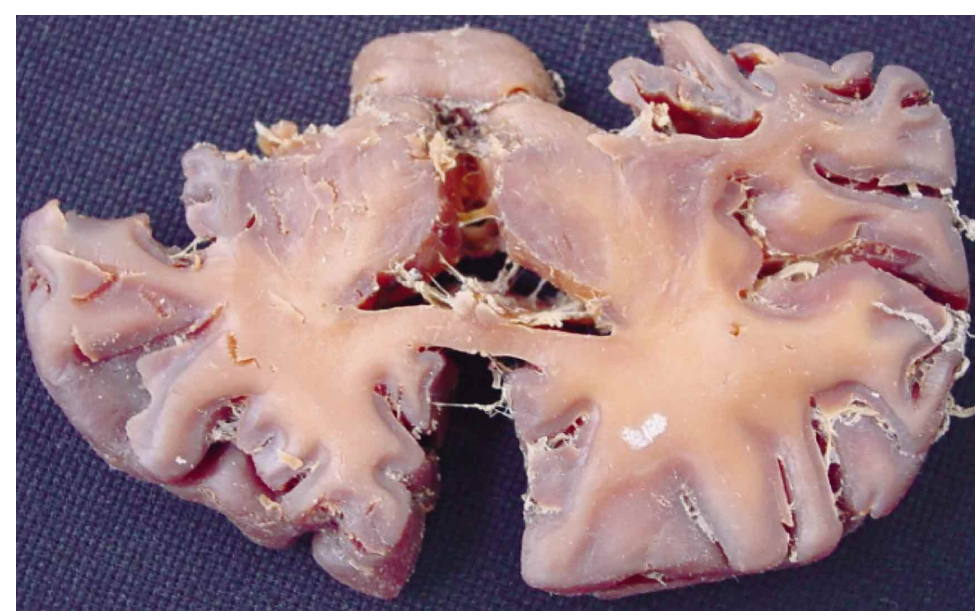

Fig. 1. Pieza de encéfalo reseco y de color oscuro por factores ambientales utilizada para aplicar la técnica para recuperación.

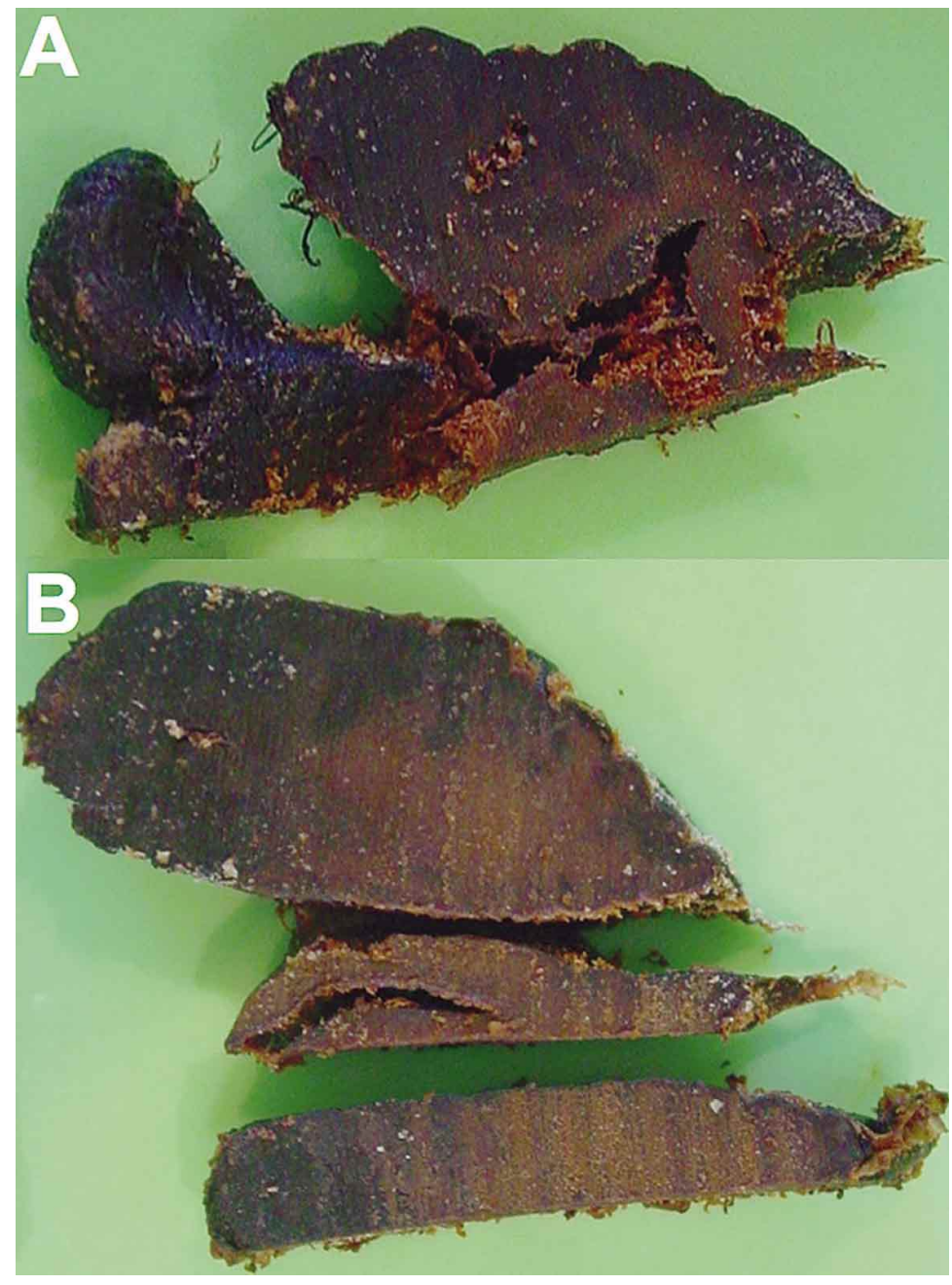

Fig. 2. Piezas de encéfalo obtenidos directamente de un cadáver con larga data de fallecido resecas y de color oscuro utilizadas para aplicar la técnica para recuperación. 


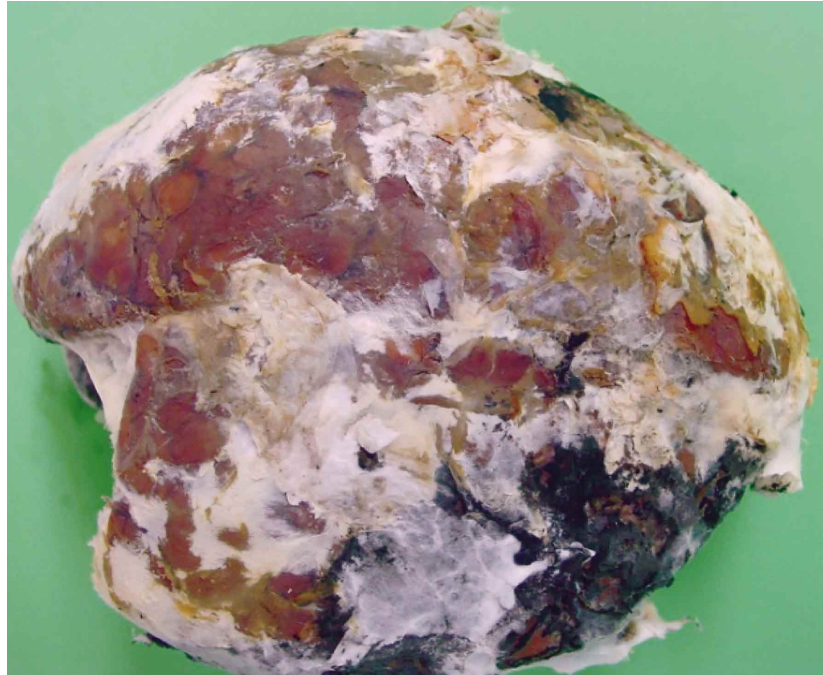

Fig. 3. Pieza encefálica contaminada por hongos utilizada para aplicar la técnica para recuperación.

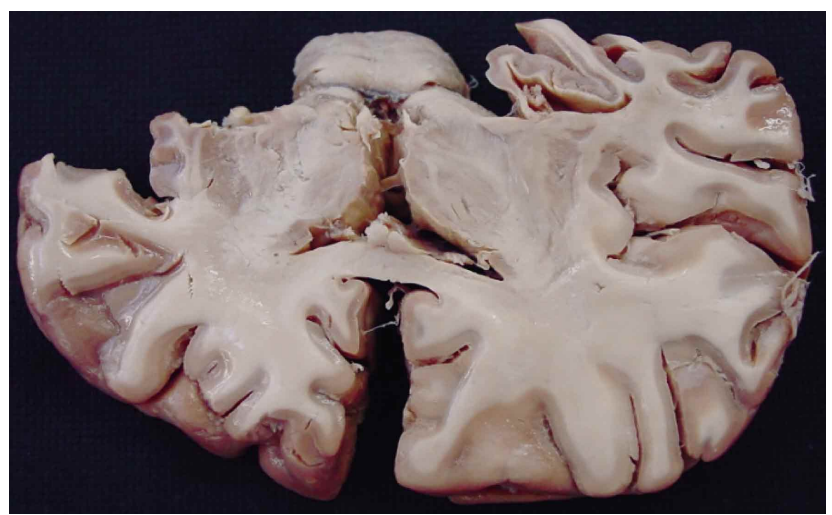

Fig. 4. Pieza encefálica expuesta a factores ambientales recuperada.

\section{DISCUSIÓN}

La técnica utilizada resultó ser un método de fácil aplicación, bajo costo y que permite recuperar muestras que estaban prácticamente desechadas (técnica sencilla), algo a tener en cuenta si consideramos lo difícil que resulta conseguir material de este tipo y particularmente que sea fresco.

$\mathrm{Al}$ decidir el protocolo de procedimiento de recuperación de las muestras, se optó por reforzar la rehidratación del grupo B, con respecto al grupo A, debido a que las muestras del primero, se encontraban notoriamente más deshidratadas que las del segundo.

Finalmente se determinó el uso de agua destilada en los procedimientos de rehidratación de las muestras de los grupos A y B, en desmedro del agua corriente, para evitar algunas posibles alteración por efecto de los otros compo-

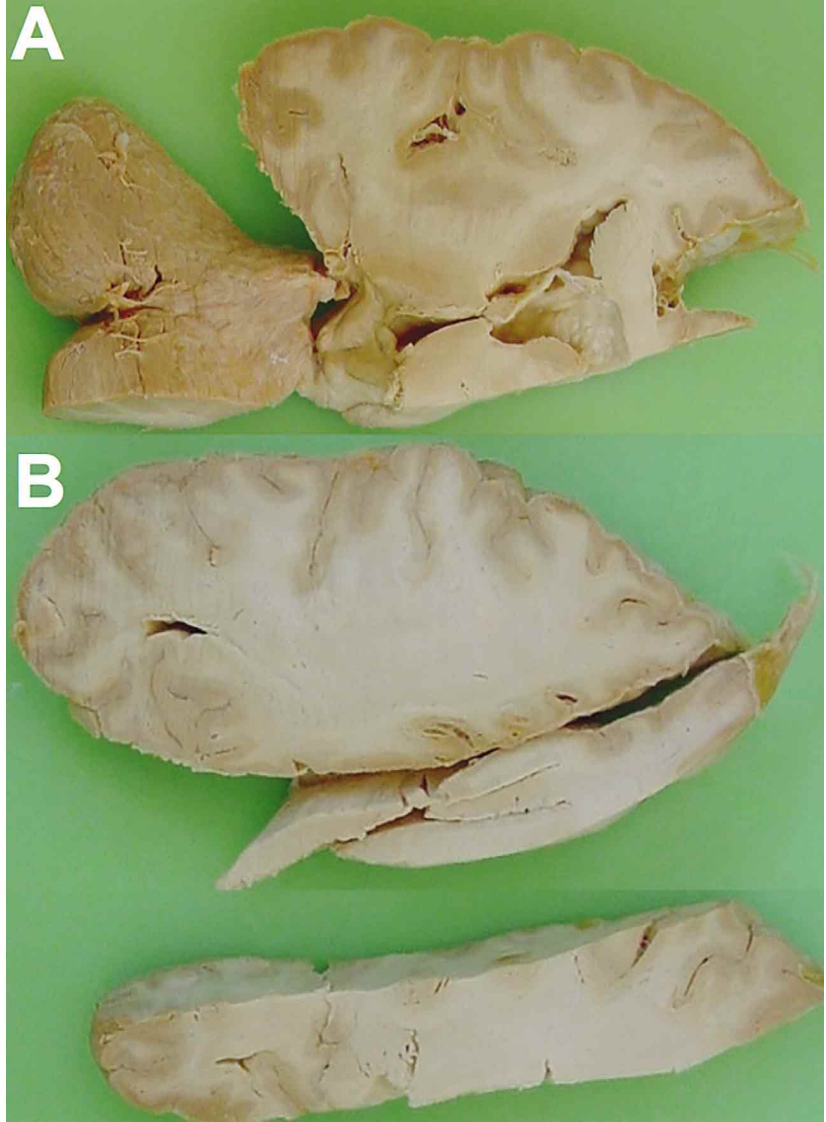

Fig. 5. Pieza encefálica obtenida de un cadáver antiguo recuperada.

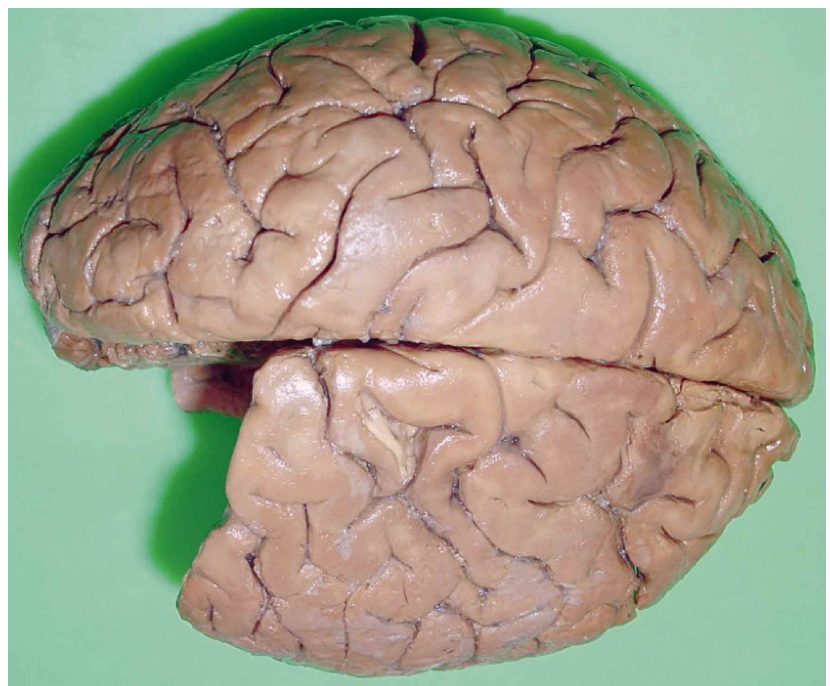

Fig. 6. Pieza encefálica contaminada por hongos recuperada.

nentes químicos presentes en ella (cloro, flúor, carbonatos de calcio y magnesio) basado en el resultado de pruebas previas que sugirieron descartarla en el protocolo final, aunque el agua corriente, sí fue utilizada para la solución de formalina. 
El motivo por el cual en las muestras del grupo $\mathrm{C}$, se refuerza y prolonga el tiempo de fijación en solución de formalina al $10 \%$, pese a ser cuestionada por ser una sustancia tóxica y con efectos cancerígenos para el usuario (Wolff $e t$ al. ), se debe a que, la acción de los hongos había provocado mayor labilidad en éstas (característica que no se aprecia en las de los grupos A y B) y, considerando las ventajosas cualidades fijadoras de esta sustancia en tejido nervioso, es que se determina tal acción.

En la generalidad de las piezas tratadas se observó una notable mejoría, con recuperación de color y consistencia, y particularmente muy evidente visualmente en las muestras del grupo $\mathrm{B}$, teniendo en consideración, sin embargo, que son piezas fijadas con formalina, la que a priori, le da un color particular.
Existe una óptima relación costo vs. beneficio, puesto que el precio de los materiales es accesible y el rendimiento que con las soluciones se logra, hacen que este método sea una real y beneficiosa alternativa de optimización del material anatómico de que se disponga.

Lo anteriormente expuesto, nos permite proponer esta técnica como tratamiento de recuperación de piezas anatómicas de sistema nervioso central en base a los esquemas protocolizados para cada tipo de muestra.

AGRADECIMIENTOS. Al Dr. Felipe Cáceres M. y a Gerardo Aguilar Z., por su generosa colaboración en la revisión de este artículo.

VALENZUELA, O. M. \& SOTO, N. R. Recovery method for anatomical pieces from the central nervous system Int. J. Morphol., 32(2):571-574, 2014.

SUMMARY: The anatomical material of the central nervous system is increasingly difficult to obtain. Despite being fixed, is very labile and when exposed to various environmental conditions during teaching, becomes deteriorated and drier, acquiring a rigid consistency and darker appearance, which eventually makes it difficult to recognize structures. At the same time, when we obtain a piece of a long-standing cadaver, we can come across a brain so dry, that its state does not provide any use. The aim of this technique was to recover these samples so they can be conveniently used for anatomical studies. We used various brain segments, including some contaminated with fungi and others obtained from old cadavers. The materials used were, hydrogen peroxide, distilled water, formaldehyde and plastic containers. We begin by manually cleaning the samples from any dust and foreign bodies that could be found on their surface. Then, continues with hydrogen peroxide baths, interspersing with re-hydration in distilled water, until we obtain the desired color and texture that allows us to macroscopically distinguish the structures. Subsequently, we reinforce fixation by immersing in formaldehyde. Subsequently, we keep them moistened with this fixation agent in sealed plastic bags indefinitely, inside covered plastic boxes. Other samples were subsequently plastinated. After we applied this technique, most of the samples were noticeably recovered, allowing recognition of structures that, previously because of their deterioration, were impossible to see. In conclusion, this method allows the recovering and gives use to samples that had been previously discarded.

KEY WORDS: Central nervous system samples; Hydrogen peroxide; Re-Hydration; Recovery.

\section{REFERENCIAS BIBLIOGRÁFICAS}

Bertone, V.; Blasi, E.; Ottone, N. \& Domínguez, M. Método de Walther Thiel para la Preservación de Cadáveres con Mantenimiento de las Principales Propiedades Físicas del Vivo. Rev. Arg. Anat. Online, 2(3):89-92, 2011.

Valdés, F.; Vega, E. \& Valenzuela, M. Two Different Plastination Techniques Comparative Study. Int. J. Morphol., 28(3):7836,2010

Valenzuela O. M.; Azocar S.C.; Werner F.K.; Vega P. E.; Valdés G. F. Plastination Experience in Polyester Resin (P-4). Int. J. Morphol., 30(3):810-3, 2012.

Wolff, D.; Villa, P.; Neirreitter, A.; Ruibal, C.; Ugon, G. A.; Salgado, G. \& Cantín, M. Comparative Study between Conservative Solutions with and without Formaldehyde in Human Placenta. Int. J. Morphol., 30(2):432-8, 2012.
Dirección para Correspondencia:

Marcos Valenzuela O.

Monseñor Álvaro del Portillo 12455

Las Condes, Santiago

CHILE

Email: marcosv@uandes.cl

Recibido: 19-07-2013

Aceptado: 27-03-2014 